\title{
AN INTRODUCTION TO THE EPR-CHAMELEON EXPERIMENT
}

\author{
LUIGI ACCARDI, KENTARO IMAFUKU, MASSIMO REGOLI \\ Centro Vito Volterra, Università di Roma "Tor Vergata" \\ E-mail: accardi@volterra.mat.uniroma2.it, \\ WEB page: http://volterra.mat.uniroma2.it
}

\begin{abstract}
On September 27 (2001), as a side activity to the "Japan-Italy Joint workshop on: Quantum open systems and quantum measurement", the first public demonstration of the dynamical EPR-chameleon experiment was performed at Waseda University in order to give an experimental answer to a long standing question in the foundations of quantum theory: do there exist classical macroscopic systems which, by local independent choices, produce sequences of data which reproduce the singlet correlations, hence violating Bell's inequality?

The EPR-chameleon experiment gives an affirmative answer to this question by concretely producing an example of such systems in the form of three personal computers which realize a local deterministic dynamical evolution whose mathematical structure is very simple and transparent.

In the experiment performed on September 27 the local dynamics used was not a reversible one because the interaction with the degrees of freedom of the apparatus was integrated out giving rise to an effective Markovian dynamics which, although mapping probability measures into probability measures, did not preserve the \pm 1 -values of the spin (or polarization) observables.

This feature was criticized by some of the partecipants and the following two questions arose:

i) is it possible to prove that the Markovian evolution, used in the experiment, is indeed the reduced evolution of a bona fide reversible evolution?

ii) if the answer to question (i) is affirmative, is it possible to reproduce the EPR correlations by simply considering empirical averages of \pm 1 -values, as one does in usual EPR type experiments?

An affirmative answer to these questions was given in the paper [AcImRe01] and it is briefly reviewed in what follows.
\end{abstract}

\section{Introduction}

In the past 20 years quantum probability has challanged the widespread belief that classical macroscopic systems cannot, by local independent choices, produce sequences of data whose correlations violate Bell's inequality.

The possibility of such a violation is not a matter of interpretation, but of fact: "local independent choices" means that two separated and non communicating experimenters make measurements but one does not know what the other measures (or even if the other one measures something); correlations 
are evaluated by means of standard procedures.

There is no space for verbal compromise in this question: either the supporters of Bell's thesis or those of quantum probability are wrong. Tertium non datur.

The goal of the present experiment is to prove that the point of view of quantum probability is correct.

On the other hand, since the Bell thesis is widely known and the quantum probability thesis - widely unknown, it might be useful to quickly pinpoint the main critiques quantum probability moves to Bell's analysis.

It is interesting to notice that while, in the past years, a multiplicity of books and papers have appeared, which reproduce modulo inessential variants, the original Bell argument, the problem to reply to the quantum probability critiques has not been tackled.

In absence of such a reply, the only reasonable conclusion is that the quantum probability critiques are sound and that the theoretical analysis, from which the contradiction between quantum theory, locality and reality is deduced, is effectively a weak one. The present experiment brings this theoretical analysis to its logical conclusion by producing a concrete counterexample.

The reason why the interpretation of quantum mechanics has been one of the most fundamental conceptual problems of modern science and practically all the best physicists, philosophers of science and mathematicians of the past century confronted themselves with this problem, is probably the uneasy feeling that there might be a deeply rooted contradiction between two of the basic pillars of contemporary physics: quantum theory and relativity theory.

For almost 40 years arguments based on violation of Bell's inequality have been deemed to provide the basis for an experimental proof of such contradiction (with the meager consolation that this contradiction might not be exploitable for human telecommunications).

The present experiment shows that this is not the case: in no way the EPR correlations and related experiments can be considered as a support of the incompatibility of quantum theory with local realistic theories, in particular relativity.

\section{The Bell inequality}

In this section we briefly survey the quantum probabilistic analysis of Bell's inequality.

Bell's inequality was proved in the paper [Be64]. In this paper, while the thesis, i.e. the inequality itself, is clearly stated and correctly proved, the mathematical assumptions from which the thesis follows (and without which

2002'0518: submitted to World Scientific on February 12, 2003 
the thesis cannot be proved) were not formulated.

This opened a debate whose goal was to try and establish which these assumptions effectively were. The reader interested in having an idea of the arguments used before quantum probability may consult the famous [Wig70] or [Stap82] or, for the connections with probability [SuZa76].

The mathematical formulation, now commonly adopted, of the Bell inequality was first given in [Ac81]. The main result of this paper consists in having realized that the mathematical assumptions on which the validity of the inequality depends are only the following two ones:

(i) that the random variables take values in the interval $[-1,1]$ (originally Bell considered only the set $\{-1,1\}$ but shortly after he extended his result to the full interval)

(ii) that all the random variables are defined on a single probability space.

More precisely:

Lemma (1) Let $A, B, C$ be random variables defined on the same probability space $(\Omega, \mathcal{F}, P)$ and with values in the set $\{-1,1\}$. Denote

$$
\langle A B\rangle:=\int_{\Omega} A(\omega) B(\omega) P(d \omega)
$$

the correlations (mean zero can be assumed without loss of generality). Then the following inequality holds:

$$
|\langle A B\rangle-\langle C B\rangle| \leq 1-\langle A C\rangle
$$

Remark The proof below is, modulo notational variations, Bell's original one. For generalizations and variants, cf. [AcRe00b].

Proof. Since the expectation value is linear

$$
|\langle A B\rangle-\langle C B\rangle|=|\langle A B-C B\rangle|
$$

Now we use $|\langle X\rangle| \leq\langle|X|\rangle$ and the fact that $A, B, C$ are \pm 1 -valued to deduce that

$$
\leq\langle|A B-C B|\rangle=\langle|A B| \cdot|1-A C|\rangle=\langle|1-A C|\rangle=\langle(1-A C)\rangle=1-\langle A C\rangle
$$

In the original Bell's inequality (1) the observable $C$ is measured by experimenter 1 in the second experiment and by experimenter 2 in the third. Thus, in order to verify violation of this inequality there must be a preliminary agreement between the two experimenters (or recourse to random choices with a posteriori matching).

The following corollary of (1) (which is an equivalence for \pm 1 -values observables), due to Clauser, Horne, Shimony, Holt (CHSH), allows to perform the violation experiments without exchange of informations on the random

2002'0518: submitted to World Scientific on February 12, 2003 
variable $C$ (typically this represents a spin or polarization direction). For this reason, even if mathematically it does not add anything to Bell's argument, it is widely used in the literature.

Corollary (2) Let $A, B, A^{\prime}, B^{\prime}$ be random variables defined on the same probability space $(\Omega, \mathcal{F}, P)$ and with values in the set $\{-1,1\}$. Then the following inequality holds:

$$
\left|\langle A B\rangle-\left\langle A^{\prime} B\right\rangle+\left\langle A B^{\prime}\right\rangle+\left\langle A^{\prime} B^{\prime}\right\rangle\right| \leq 2
$$

Proof. With the replacements $B \rightarrow B^{\prime}, C \rightarrow-C$, (1) becomes

$$
\left|\left\langle A B^{\prime}\right\rangle+\left\langle C B^{\prime}\right\rangle\right|=1+\langle A C\rangle
$$

Adding (1) to (5) and replacing $C$ by $A^{\prime}$ we get

$$
\left|\langle A B\rangle-\left\langle A^{\prime} B\right\rangle\right|+\left|\left\langle A B^{\prime}\right\rangle+\left\langle A^{\prime} B^{\prime}\right\rangle\right| \leq 2
$$

which implies (4).

The following rephrasing of Corollary (2) is used to establish a connection between Bell's inequality and the predictions of quantum mechanics.

Corollary (3). Let $S_{a}^{(1)}, S_{c}^{(1)}, S_{b}^{(2)}$ be \pm 1 -valued random variables defined on a probability space $(\Omega, \mathcal{F}, P)$. Then

$$
\left|\left\langle S_{a}^{(1)} S_{b}^{(2)}\right\rangle-\left\langle S_{c}^{(1)} S_{b}^{(2)}\right\rangle\right| \leq 1-\left\langle S_{a}^{(1)} S_{c}^{(1)}\right\rangle
$$

Proof. (6) is obtained by replacing, in (1):

$$
A \rightarrow S_{a}^{(1)} \quad ; \quad B \rightarrow S_{b}^{(2)} \quad ; \quad C \rightarrow S_{c}^{(1)}
$$

Remark. Notice that, in a quantum mechanical context, (6) would not be measurable for $a \neq c$.

Remark. The CHSH analogue of (6) is

$$
\left|\left\langle S_{a}^{(1)} S_{b}^{(2)}\right\rangle-\left\langle S_{a^{\prime}}^{(1)} S_{b}^{(2)}\right\rangle+\left\langle S_{a}^{(1)} S_{b^{\prime}}^{(2)}\right\rangle+\left\langle S_{a^{\prime}}^{(1)} S_{b^{\prime}}^{(2)}\right\rangle\right| \leq 2
$$

With these notations the main mathematical conclusion, used in the comparison of the predictions of Bell's inequality with those of quantum theory is the following

Theorem (4). There cannot exist a stochastic process

$$
S_{a}^{(1)}, S_{b}^{(1)} \quad a, b \in[0,2 \pi]
$$

defined on a probability space $(\Omega, \mathcal{F}, P)$ and with values in the set $\{ \pm 1\}$, whose correlations are given by:

$$
\left\langle S_{a}^{(1)} S_{b}^{(2)}\right\rangle=-\cos (a-b) \quad ; \quad a, b \in[0,2 \pi]
$$


Remark. According to quantum theory the expression in the right-hand side of (8) is the correlation of two spin or polarization observables, along directions $a, b$, of two quantum particles in singlet state. These correlations have been experimentally confirmed by many experiments since the early days of quantum mechanics.

Proof. Suppose, by contradiction, that such a process exists. Then, by Corollary (3), it must satisfy (6). A corollary of (8) is the singlet condition:

$$
\left\langle S_{a}^{(1)} S_{b}^{(2)}\right\rangle=-1
$$

which is equivalent to

$$
S_{a}^{(1)}=-S_{a}^{(2)} \quad P-\text { a.e. }
$$

The singlet condition (9) implies that (6) is equivalent to

$$
\left|\left\langle S_{a}^{(1)} S_{b}^{(2)}\right\rangle-\left\langle S_{c}^{(1)} S_{b}^{(2)}\right\rangle\right| \leq 1+\left\langle S_{a}^{(1)} S_{c}^{(1)}\right\rangle
$$

But, choosing

$$
a=0 \quad ; \quad b=\pi / 2 \quad ; \quad c=\pi / 4
$$

one sees that (10) leads to the contradiction $\sqrt{2} \leq 1$.

Remark. The deduction of (7) (i.e. CHSH) does not require the singlet condition (9). However once a single probability space is postulated, the quantum mechanical condition (8) implies (9).

\section{The implications of Bell's inequality according to Bell}

Theorem (4) above synthetizes the conclusions of the quantum probabilistic analysis of the implications of Bell's inequality.

Bell's conclusions about these implications were quite different! They are clearly stated both in the Introduction and in the Conclusions of [Be64] and can be summarized by Bell's own words:

"... In a theory in which parameters are added to quantum mechanics to determine the results of individual measurements, without changing the statistical predictions, there must be a mechanism whereby the setting of one measuring device can influence the reading of another instrument, however remote. Moreover the signal involved must propagate instantaneously so that the theory could not be Lorentz invariant. ..." (cf. the beginning of Section VI of [Be64]).

A theory "... in which parameters are added to quantum mechanics to determine the results of individual measurements, without changing the

2002'0518: submitted to World Scientific on February 12, 2003 
statistical predictions ..." is called a "hidden variable theory". Such a theory is also called "realistic" because in it observables have always a definite value, as opposed to the orthodox interpretation of quantum mechanics in which observables have virtual values which become actual by virtue of the measurement.

To "... add parameters to quantum mechanics ..." means that the quantum observables are represented as functions on a single space $\Omega$ (the space of hidden parameters).

"... without changing the statistical predictions ..." means (in the EPR context) that the hidden parameters are distributed according to a (possibly unknown) probability measure $P$ on $\Omega$ and that the experimentally measurable correlations of any pair of observables $A, B$ are those predicted by classical probability, i.e.

$$
\int_{\Omega} A(\omega) B(\omega) P(d \omega)
$$

The locality condition, according to Bell, is that:

"... the result $B$ for particle 2 does not depend on the setting $a$, of the magnet for particle 1, nor $A$ on $b$. ..." (cf. Section II of [Be64]. In fact at this point Bell quotes EPR.).

A hidden variable theory, satisfying the above conditions is called a local realistic model for the EPR (singlet) correlations.

For these reasons Bell's conclusion on the implications of his inequality is often synthetized in the statement:

"... A local realistic model for the EPR correlations cannot exist. ..."

Bell's statement reported above has given rise to a huge literature turning around the apparent dichotomy pointed out in this statement: if we insist on a realistic point of view, then we have to give up locality and conversely, if we insist on locality, then we have to give up a realistic point of view. Both choices would be heavy for a physicist.

The quantum probabilistic approach offers to the physicists a way out by criticizing Bell's analysis and proving that:

i) the contradiction, pointed out by Bell, arises only from his implicit postulate that 3 statistical correlations, coming from 3 mutually incompatible experiments, can be described within a single classical probabilistic model

ii) that this implicit postulate is by no means a consequence of locality and reality.

If this implicit postulate is not assumed, then Bell's proof is at fault already in its first step, i.e. (2), because if we write explicitly the identity

$$
\langle A B\rangle-\langle C B\rangle=\langle A B-C B\rangle
$$

2002'0518: submitted to World Scientific on February 12, 2003 
we find

$$
\begin{array}{r}
\int_{\Omega} A(\omega) B(\omega) P_{a, b}(d \omega)-\int_{\Omega} C(\omega) B(\omega) P_{c, b}(d \omega) \\
=\int_{\Omega}(A(\omega) B(\omega)-C(\omega) B(\omega)) P_{a, c, b}(d \omega)
\end{array}
$$

and, while the pair joint probabilities $P_{a, b}, P_{c, b}, \ldots$ are experimentally observable, there is no reason to postulate, as Bell implicitly does when using this formula, that the, experimentally unobservable, triple joint probabilities $P_{a, c, b}$ exist.

It is well known from classical probability that there are constraints, i.e. compatibility conditions, which relate the pair with the triple joint probabilities and which are necessary conditions for the existence of the latter ones. Since the pair correlations are deduced from the pair probabilities and since, when using (2), Bell is postulating a priori the existence of these (experimentally unobservable) triple joint probabilities, the only rational conclusion he can draw from his argument is that the inequality (1) (Bell's inequality) is one of these necessary conditions.

This was the critique that, starting from 1981 [Ac81], quantum probability opposed to Bell's argument.

One might try to counter this critique by arguing that the existence of the triple probabilities is a consequence of the "realism" assumption.

For example suppose that in a box there are many pairs of balls whose color can be either green or brown. Moreover each ball is either made of glass or of wood and it weights either 10 or 20 grams. The rules of the game are such that you can only measure one observable at the time on each ball (color, weight, material). Thus on each pair we can simultaneously measure at most two observables and we can make an experimental analysis of the joint statistics of all possible pairs of observables ("color-material", "color-weight", $\ldots)$.

Because of the rules of the game the triple joint probabilities "colormaterial-weight" are not accessible to experiment.

However the "realism assumption" tells us that any one of the possible combinations (color, material, weight) has a definite relative frequency in the box and that the pair statistics we observe, is a consequence of this (unobservable) triple statistics.

Arguments of this kind are quite reasonable: for example they are at the basis of classical statistical mechanics and it is probable that Einstein had in mind something of this kind when speaking of "objective reality".

According to quantum probability there is a more subtle notion of "objec-

2002'0518: submitted to World Scientific on February 12, 2003 
tive reality" which gives a better intuition of the behavior of quantum systems (but it by no means restricted to them). We call the corresponding realism "chameleon realism" as opposed to the "ballot box realism" of classical statistical mechanics.

Suppose that, in the above example, you leave the rules of the game unaltered, but you replace the pairs of balls by pairs of chameleons and the observables (color, material, weight) by (color on the leaf, color on the wood, weight).

Is it still reasonable to believe that the pair statistics you observe, is a consequence of some (unobservable) triple statistics?

A little thought shows that the answer is: No!

According to quantum probability, quantum systems are much more similar to chameleons (adaptive: we measure the response to an interaction) than to balls (passive: we read what was in the box).

\section{The chameleon effect}

The attempt to translate in a precise mathematical and physical language the intuitive difference between "ballot boxes" and "chameleons" leads to a natural generalization of von Neumann's measurement theory.

The generalization consists in introducing, in this theory, the notions of locality and causality.

It is widely accepted, since von Neumann's original analysis, that a qualitative analysis of the measurement process should start from the joint (unitary) evolution $u_{S, A}$ (system, apparatus): for simplicity we consider discrete time. Thus, if $\psi_{o}$ is the initial state of the system, its state at the time of measurement is

$$
\psi:=\psi_{o} \circ u_{S, A}
$$

Now suppose that we want to measure the observable $S_{a}=S_{a} \otimes 1_{M}$ of the system (say: spin in direction $a$ ). Then the apparatus must be prepared to measure $S_{a}$ (say: by orienting a magnetic field in direction $a$ ). Therefore the interaction Hamiltonian between system and apparatus, hence also the joint dynamics, will depend on $a$ :

$$
u_{S, A}:=u_{S, A(a)}:=u_{a}
$$

In other words: the dynamics of a system depends on the observable we want to measure: this is the chameleon effect. As anybody can see, it is a simple 
corollary of the standard ideas on measurement theory.

Now suppose that the system, hence the apparatus, is made up of two spatially separated parts: $\left(1,2, A_{1}, A_{2}\right)$ and that we measure independently

$$
S_{a}^{(1)}=\left(S_{a} \otimes 1_{2}\right) \otimes 1_{M} \quad\left(\text { resp. } S_{b}^{(2)}=\left(1_{1} \otimes S_{b}\right) \otimes 1_{M}\right)
$$

on particle 1 (resp. particle 2). Then, according to the chameleon effect, we will have

$$
u_{S, A}:=u_{a, b}
$$

and, according to quantum (or classical) mechanics, the pair correlations will be

$$
\left\langle S_{a}^{(1)} S_{b}^{(2)}\right\rangle:=\psi_{o} \circ u_{a, b}\left(S_{a}^{(1)} S_{b}^{(2)}\right)=\psi_{a, b}\left(S_{a}^{(1)} S_{b}^{(2)}\right)
$$

This shows that the pair joint probability $P_{a, b}$, corresponding to these correlations, depends on $a, b$, hence the application of Bell's inequality is impossible.

However, by considering the mean value of a single particle observable, say $S_{a}^{(1)}$ :

$$
\psi\left(S_{a}^{(1)}\right):=\psi_{o}\left(u_{a, b}\left(S_{a}^{(1)}\right)\right)
$$

we see that, for a general dynamics, the mean value of an observable of particle 1 will depend on the measurement we do on particle 2: this means that the EPR locality condition is not satisfied.

If we want it to be satisfied, we have to restrict the class of allowed dynamics and also the class of allowed initial states.

The physical arguments which allow to define such restrictions have been discussed in previous papers of the authors (cf. [AcRe01a] for bibliography).

The EPR locality condition is mathematically expressed by

$$
u_{a, b}:=u_{a} \otimes u_{b}
$$

and the causality condition by

$$
\psi_{o}:=\psi_{1,2} \otimes \psi_{A_{1}} \otimes \psi_{A_{2}}
$$

where both $\psi_{A_{1}}, \psi_{A_{2}}$ may depend on state of the system 1 (resp.2). With these restrictions one easily computes that the EPR locality condition is satisfied. However (8) and (6) show that the pair joint probabilities, corresponding to pair correlations, still depend on $a, b$, hence the application of Bell's inequality is still impossible. 
This extension of the standard quantum theory of measurement was first proposed in [Ac93]. The experiment discussed in the present conference is a concrete realization of this abstract scheme.

\section{Description of the dynamical model}

In the present section we construct a dynamical system which simulates locally the EPR correlation (8).

In the idealized dynamical system considered in our experiment we consider only two time instants 0 (initial) and 1 (final) so, in our case, a "trajectory" consists of a single jump. We do not describe the space-time details of the trajectory because we are only interested in distinguishing 2 cases:

- at time 1 the particle is in the apparatus (and in this case it is detected with certainty)

- at time 1 the particle is not in the apparatus (and in this case it makes no sense to speak of detection)

Thus our "configuration space" for the single particle will be made of 3 points: $s$ (source), 1 (inside apparatus), 0 (outside apparatus). Since at time 0 the "position" of both particles is always $s$, because of the chameleon effect, the position $q_{j, 1}$ of particle $j=(1,2)$ at time 1 will depend on the polarization $a_{j}$, on the inner state $\sigma$ and on the state $\lambda_{j}$ of the apparatus $M_{j}(j=1,2)$ :

$$
q_{j, 1}=q_{j, 1}\left(a_{j}, \sigma, \lambda_{j}\right) \quad ; \quad j=1,2,
$$

The local, deterministic dynamical law of this dependence is described as followed.

1. The state space of the composite system (particles, apparatus) is

$$
\begin{aligned}
\{\text { position space }\} & \times\{\text { inner state space }\} \times\{\text { apparatus space }\} \\
= & \{s, 0,1\} \times[0,2 \pi]^{2} \times[0,1]^{2}
\end{aligned}
$$

2. The initial state is always of the form

$$
\left(s, s, \sigma_{1}, \sigma_{2}, \lambda_{1}, \lambda_{2}\right) \in\{s\}^{2} \times[0,2 \pi]^{2} \times[0,1]^{2}
$$

i.e. the initial position of both particles is always $s$. 
3. To speak of correlations only makes sense if the deterministic trajectories of both particles end up in the detectors (pre-determination). This means that the statistics is conditioned to the subset

$$
\{1\}^{2} \times[0,2 \pi]^{2} \times[0,1]^{2}
$$

of the state space.

4. Just by changing the order of the factors the state space can be realized as

$$
\begin{aligned}
& \left\{\left(s_{1}, \sigma_{1}, \lambda_{1} ; s_{2}, \sigma_{2}, \lambda_{2}\right)\right\} \\
& \quad \in(\{s, 0,1\} \times[0,2 \pi] \times[0,1]) \times(\{s, 0,1\} \times[0,2 \pi] \times[0,1])
\end{aligned}
$$

Therefore a local deterministic dynamics is uniquly determined by the assignment of two functions $T_{1, a}, T_{2, b}$ :

$$
\begin{gathered}
\left(s_{1}, \sigma_{1}, \lambda_{1} ; s_{2}, \sigma_{2}, \lambda_{2}\right) \rightarrow\left(T_{1, a}\left(s_{1}, \sigma_{1}, \lambda_{1}\right), T_{2, b}\left(s_{2}, \sigma_{2}, \lambda_{2}\right)\right) \\
=:\left(q_{1, a}\left(s_{1}, \sigma_{1}, \lambda_{1}\right), s_{1, a}\left(s_{1}, \sigma_{1}, \lambda_{1}\right), m_{1, a}\left(s_{1}, \sigma_{1}, \lambda_{1}\right) ;\right. \\
\left.q_{2, b}\left(s_{2}, \sigma_{2}, \lambda_{2}\right), s_{2, b}\left(s_{2}, \sigma_{2}, \lambda_{2}\right), m_{2, b}\left(s_{2}, \sigma_{2}, \lambda_{2}\right)\right)
\end{gathered}
$$

Moreover it is convenient to identify the endpoints of both intervals $[0,2 \pi]$ and $[0,1]$, i.e. to identify these intervals to circles so that the functions $q_{j, x}, s_{j, x}, m_{j, x}(j=1,2, x=a, b)$, as functions of the variables $\sigma, \lambda$ can be extended by periodicity to the whole real line (period $2 \pi$ in $\sigma$, period 1 in $\lambda$ ). This allows to give a meaning to formula (17) of [AcImRe01] in full generality, i.e. without appealing to special choice (11) of [AcImRe01].

5 . With these conventions, for every $a, b \in[0,2 \pi]$, a deterministic dynamics as follows

$$
\left(s_{1}, \sigma_{1}, \lambda_{1} ; s_{2}, \sigma_{2}, \lambda_{2}\right) \mapsto\left(q_{1, a}\left(s_{1}, \sigma_{1}, \lambda_{1}\right), \sigma_{1} ; q_{2, b}\left(s_{2}, \sigma_{2}, \lambda_{2}\right), \sigma_{2}, \lambda_{2}\right)
$$

i.e. the inner state of the particle and of the apparatus do not vary under the evolution, but the position varies according to the law:

$$
\begin{gathered}
q_{1, a}\left(s, \sigma_{1}, \lambda_{1}\right)=\chi_{\left[0, p_{1, a}\left(\sigma_{1}\right)\right]}\left(\lambda_{1}\right), \quad \chi_{[0, x]}(\lambda)=\left\{\begin{array}{l}
1, \text { for } \lambda \in[0, x] \\
0, \text { for } \lambda \notin[0, x]
\end{array}\right. \\
q_{2, b}\left(s, \sigma_{2}, \lambda_{2}\right)=1 \\
p_{1, a}\left(\sigma_{1}\right)=\frac{1}{4}\left|\cos \left(\sigma_{1}-a\right)\right|
\end{gathered}
$$

Remember that the initial position of both particles is always $s$. Therefore it is sufficient to define the dynamics only in this case. 
6. For every setting $(a, b) \in[0,2 \pi]^{2}$ of the apparata, the initial probability distribution of our deterministic dynamical system is given by:

$$
\begin{gathered}
\delta_{s, s_{1}} \delta_{s, s_{2}} \frac{1}{2 \pi} \delta\left(\sigma_{1}-\sigma_{2}\right) \delta\left(m_{1, a}\left(\sigma_{1}, \lambda_{1}\right)-m_{a}\right) \\
\times \delta\left(m_{2, b}\left(\sigma_{2}, \lambda_{2}\right)-m_{b}\right) d \sigma_{1} d \sigma_{2} d \lambda_{1} d \lambda_{2}
\end{gathered}
$$

where $m_{a}, m_{b}$ are fixed numbers in $[0,1]$ and

$$
\begin{gathered}
m_{1, a}\left(\sigma_{1}, \lambda_{1}\right)=\frac{4 \lambda_{1}}{\sqrt{2 \pi}\left|\cos \left(\sigma_{1}-a\right)\right|} \\
m_{2, b}\left(\sigma_{2}, \lambda_{2}\right)=\frac{\lambda_{2}}{\sqrt{2 \pi}}
\end{gathered}
$$

Finally the random variables $S_{a}^{(1)}, S_{b}^{(2)}$ :

$$
\{s\}^{2} \times[0,2 \pi]^{2} \times[0,1]^{2} \rightarrow\{ \pm 1\}
$$

are defined by

$$
S_{a}^{(1)}(s, \sigma, \lambda)=\operatorname{sgn}(\cos (\sigma-a)) ; S_{a}^{(2)}=-S_{a}^{(1)}
$$

It is now a matter if simple calculations (cf. section (2) of [AcImRe01]) to verify that the correlations

$$
\left\langle S_{a}^{(1)} S_{b}^{(2)}\right\rangle=\int_{\Omega} S_{a}^{(1)}\left(T_{1, a}\left(s, \sigma_{1}, \lambda_{1}\right)\right) S_{b}^{(2)}\left(T_{2, b}\left(s, \sigma_{2}, \lambda_{2}\right)\right) d P_{a, b}(\omega)
$$

$\left(\omega=\left(s, \sigma_{1}, \lambda_{1} ; s, \sigma_{2}, \lambda_{2}\right)\right)$ are precisely the EPR correlations.

Finally notice that the dynamics (10) is slightly simplified with respect to the one described in [AcImRe01]. However, due to the choice (11) of [AcImRe01] this simplification does not change the calculations in the specific case under consideration. For more general classes of models the simplification (10) is convenient because with this choice the state space is mapped into itself by the dynamics and no additional identifications are required.

There is no conceptual difficulty to include in our model the consideration of the space-time trajectory of the particle. This surely would improve the present model, however the main conclusion of our experiment, i.e. the reproducibility of the EPR correlations by a classical, deterministic, local dynamical system, will not change. 


\subsection{Description of the experiment}

1. Let $N \leq N_{\text {tot }}$ be natural integers and let

$$
\left\{\sigma_{j}: j=1, \ldots, N\right\}
$$

be the sequence of numbers either deterministically or pseudo-randomly distributed in $[0,2 \pi]$ with good equidistribution properties. (cf. the option $D$ (deterministic) or $R$ (random) that has been inserted in the program of the experiment.) Let $N\left(\sigma_{j}\right)(j=1, \ldots, N)$ denote a sequence of natural integers such that

$$
\sum_{j=1}^{N} N\left(\sigma_{j}\right)=N_{t o t}
$$

Remark. $N_{\text {tot }}$ represents the (physically unobservable) "total number" of entangled pairs emitted by the source. $N\left(\sigma_{j}\right)$ is the number of times that the imput $\sigma_{j}$ is produced in the sequence (13).

2. For each $j$ from 1 to $N$, repeat the following 3 operations (a), (b), (c), $N\left(\sigma_{j}\right)$ times

(a) The central computer sends $\sigma_{j}$ to the computers 1 and 2 .

(b) Computer 1 computes the position of particle 1 using the deterministic dynamics and sends back $S_{a}^{(1)}\left(\sigma_{j}\right)(=1$ or -1$)$ if the particle is inside the apparatus. It sends back nothing if the particle is outside the apparatus. Computer 2 does the same thing. The deterministic dynamics is such that $S_{a}^{(1)}\left(\sigma_{j}\right)$ is sent back with probability $p_{1, a}\left(\sigma_{j}\right)$ and $S_{b}^{(2)}\left(\sigma_{j}\right)$ is sent back with probability $p_{2, b}\left(\sigma_{j}\right)$ where $p_{1, a}, p_{2, b}$ are sufficiently regular probability densities (say piecewise smooth with a finite number of discontinuities in $[0,2 \pi]$.

Remark. This corresponds in the real experiments, to labeling the local detection time of the photon. When both computers send back a value \pm 1 , then we say that a coincidence occurs. The emergency of these probability in a deterministic context is due to the fact that the dynamics has strong chaotic properties.

(c) Only in case of a coincidence, i.e. when the central computer receives the value \pm 1 from both computers, the central computer computes the "correlation product" $S_{a}^{(1)}\left(\sigma_{j}\right) S_{b}^{(2)}\left(\sigma_{j}\right)$. 
3. The central computer computes the correlation as

$$
\frac{\text { Sum of all correlation products }}{\text { The total number of coincidences }} \text {. }
$$

Remark. This is what is done in all experiments and a corresponds to the statement of the problem because, up to now, the EPR correlations have always been integrated as equal time correlations.

\subsection{Computation of the correlations}

Introducing

$$
p\left(\sigma_{j}\right)=\frac{N\left(\sigma_{j}\right)}{N_{t o t}}
$$

the expected number of coincidences $\mathcal{N}_{\text {coincidences }}$ and the sum of all correlation products $\mathcal{S}_{\text {correlations }}$ become respectively

$$
\begin{aligned}
\mathcal{N}_{\text {coincidences }} & =\sum_{j=1}^{N} N\left(\sigma_{j}\right) p_{1, a}\left(\sigma_{j}\right) p_{2, b}\left(\sigma_{j}\right) \\
& =N_{\text {tot }} \sum_{j=1}^{N} p\left(\sigma_{j}\right) p_{1, a}\left(\sigma_{j}\right) p_{2, b}\left(\sigma_{j}\right) \\
\mathcal{S}_{\text {correlations }} & =\sum_{j=1}^{N} N\left(\sigma_{j}\right) p_{1, a}\left(\sigma_{j}\right) p_{2, b}\left(\sigma_{j}\right) S_{1}^{(a)}\left(\sigma_{j}\right) S_{2}^{(b)}\left(\sigma_{j}\right) \\
& =N_{\text {tot }} \sum_{j=1}^{N} p\left(\sigma_{j}\right) p_{1, a}\left(\sigma_{j}\right) p_{2, b}\left(\sigma_{j}\right) S_{1}^{(a)}\left(\sigma_{j}\right) S_{2}^{(b)}\left(\sigma_{j}\right)
\end{aligned}
$$

Thus the correlation defined by (14) is

$$
\frac{\mathcal{S}_{\text {correlations }}}{\mathcal{N}_{\text {coincidences }}}=\frac{\sum_{j=1}^{N} p\left(\sigma_{j}\right) p_{1, a}\left(\sigma_{j}\right) p_{2, b}\left(\sigma_{j}\right) S_{1}^{(a)}\left(\sigma_{j}\right) S_{2}^{(b)}\left(\sigma_{j}\right)}{\sum_{j=1}^{N} p\left(\sigma_{j}\right) p_{1, a}\left(\sigma_{j}\right) p_{2, b}\left(\sigma_{j}\right)}
$$

and therefore

$$
\frac{\mathcal{S}_{\text {correlations }}}{\mathcal{N}_{\text {coincidences }}} \rightarrow \frac{\int_{0}^{2 \pi} d \sigma p(\sigma) p_{1, a}(\sigma) p_{2, b}(\sigma) S_{1}^{(a)}(\sigma) S_{2}^{(b)}(\sigma)}{\int_{0}^{2 \pi} d \sigma p(\sigma) p_{1, a}(\sigma) p_{2, b}(\sigma)}
$$

where $p(\sigma)$ is a probability density with properties analogue to $p_{1, a}$ and $p_{2, b}$.

2002'0518: submitted to World Scientific on February 12, 2003 


\subsection{The EPR correlations}

In our experiment the deterministic dynamics is chosen in such a way that the emerging probabilities have the form :

$$
\begin{aligned}
& p(\sigma)=\frac{1}{2 \pi} \quad, \quad p_{1, a}(\sigma)=\frac{1}{4}|\cos (\sigma-a)| \quad, \quad p_{2, b}(\sigma)=1 \\
& S_{1}^{(a)}(\sigma)=\operatorname{sgn}(\cos (\sigma-a)), \quad S_{2}^{(b)}(\sigma)=-\operatorname{sgn}(\cos (\sigma-b))
\end{aligned}
$$

With these choices we obtain

$$
\begin{gathered}
\text { numerator of }(19)=-\frac{1}{2 \pi} \cos (a-b) \\
\text { denominator of }(19)=\frac{1}{2 \pi}
\end{gathered}
$$

Therefore for large $N$ the correlation (14) or equivalently (18), (19) is well approximated by

$$
-\cos (a-b)
$$

which is exactly the EPR correlation. We underline that, as shown by (23), even if the mechanism of coincidences depends on the setting of the apparatus, the expected number of coincidences (23) is independent of it, in agreement with the experimental results [Aspects82].

\section{References}

[Ac81] Luigi Accardi: Topics in quantum probability, Phys. Rep. 77 (1981) 169-192

[Ac93] L. Accardi:Einstein-Bohr: one all, in: The interpretation of quantum theory: where do we stand?, Acta Enciclopedica, Istituto dell'Enciclopedia Italiana (1994) 95-115; Volterra preprint N. 174 (1993).

[AcRe00b] Luigi Accardi, Massimo Regoli:

Locality and Bell's inequality.

Preprint Volterra, N. 427 (2000) quant-ph/0007005; an extended version of this paper, including the description of the present experimen will appear in the proceedings of the conference "Foundations of Probability and Physics", Vaxjo University, Sweden, November 27 - December 1 (2000), A. Khrennikov (ed.), World Scientific (2001) 
[AcRe01a] Luigi Accardi, Massimo Regoli: The EPR correlations and the chameleon effect, discussed during the "Japan-Italy Joint workshop on Quantum open systems and quantum measurement", Waseda University, 27-29 September 2001; Preprint Volterra, N. 487 (2001)

[AcImRe01] L.Accardi, K.Imafuku, M.Regoli:On the physical meaning of the EPR-chameleon experiment, Infinite dimensional analysis, quantum probability and related topics, 5 N. 1 (2002) 1-20; quant-ph/0112067; Volterra Preprint December (2001) N. 494

[Be64] Bell J.S: On the Einstein Podolsky Rosen Paradox, Physics 1 no.3. 195-200 1964.

[Stap82] Stapp H. P.:Bell's Theorem as a Nonlocality Property of Quantum Theory, Physical Review Letters, vol. 49, no. 20 (1982)

[SuZa76] Suppes P., Zanotti M.: On the Determinism of Hidden Variable Theories with Strict Correlation and Conditional Statistical Independence of Observables, Logic and Prob. in Quant. Mech., 445-455 (1976)

[Wig70] E.P. Wigner: Amer Journ. of Phys. 38 (1970) 1005

[Aspects82] Alain Aspect, Philippe Grangier, and Gerard Roger, Phys. Rev. Lett. 49, 91 (1982) 